\title{
Die Verletzlichkeit des Menschen
}

\section{Adrian Ritter}

Freischaffender Journalist

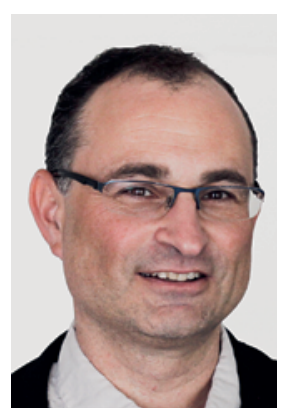

Vulnerable Patientinnen und Patienten: Als ich diesen Begriff zum ersten Mal hörte, war ich etwas irritiert. Sind nicht alle Patienten vulnerabel, weil sie ein Leiden haben? Der Ethiktag des Universitätsspitals Zürich schärfte im Februar meine Wahrnehmung des Begriffes. Die Veranstaltung war der Situation von vulnerablen Patientinnen und Patienten im Akutspital gewidmet. Dabei wurde mir klar: Nicht alle Patienten sind im selben Ausmass vulnerabel. Vulnerabilität lässt sich auf verschiedene Arten definieren. Im Kern aber geht es um Folgendes: Im Gegensatz zu «robusten» Patienten

\section{Vulnerable Patientinnen und Patienten sind zusätzlich zu ihrem Leiden verletzlich oder benachteiligt.}

mit einer bestimmten Erkrankung sind vulnerable Patientinnen und Patienten zusätzlich verletzlich oder benachteiligt - beispielsweise aufgrund ihrer Sprachkompetenz, Bildung, psychischen Verfassung, einer Behinderung oder wegen Armut.

Die Vulnerabilität von Patientinnen und Patienten zu berücksichtigen heisst, Diversität und spezifische Bedürfnisse von Menschen zu berücksichtigen. Mit diesem Anspruch hat sich eine Reihe von Schweizer Spitälern zum Netzwerk "Swiss Hospitals for Equity» (hospitals4equity.ch) zusammengeschlossen. Ihr Ziel: Für alle Patientinnen und Patienten eine qualitativ gute Gesundheitsversorgung gewährleisten. Was selbstverständlich tönt, ist bisweilen eine Herausforderung - etwa im Falle der Kommunikation mit Migrantinnen und Migranten, wo Dolmetscherdienste nötig sein können. Der Verbund der Spitäler geht denn auch auf eine europäische Initiative für migrantenfreundliche Spitäler zurück. Mit dem Begriff «Equity» will das Netzwerk aber betonen, dass nicht nur Migrantinnen und Migranten vulnerabel sein können. Armutsbetroffene Menschen suchen vielleicht aus Angst vor den Kosten gar nicht erst medizinische Hilfe auf. Studien zeigen zudem, dass gewisse Krankheiten eher als männlich oder weiblich gelten und beim anderen Geschlecht eher übersehen werden - Depressionen bei Männern und Herzinfarkte bei Frauen beispielsweise.

Wer vulnerablen Menschen gerecht werden will, muss ihre Vulnerabilität erst erkennen. Dabei ist Fingerspitzengefühl ebenso gefragt wie Fachwissen. Hilfreich sein können Instrumente in Richtung eines Vulnerabilitäts-Assessment - Ansätze dazu existieren etwa unter Begriffen wie Sozialanamnese und Belastungsbarometer.

Ist eine Vulnerabilität erkannt, gilt es, etwa in einem Spital, vorhandene Unterstützungen wie den Sozialdienst, ein Care-Team oder die Seelsorge in die Behandlung miteinzubeziehen. Analoges gilt natürlich für niedergelassene Ärzte und ihr Netzwerk. Auf Vulnerabilitäten Rücksicht zu nehmen kann ein zeitlicher Mehraufwand sein. Diesen wenn immer möglich zu leisten ist nicht nur eine Frage der Menschenwürde, sondern kann sich auch positiv auf die Behandlung auswirken. Fühlen sich vulnerable Menschen ernst genommen und unterstützt, sind sie vermutlich kooperativer bei der Suche nach Krankheitsursachen und zeigen eine höhere Therapietreue.

Das Thema Vulnerabilität findet zunehmend Beachtung in der Medizin. So sind in einzelnen Spitälern etwa eine

Vulnerabilität zu berücksichtigen ist eine Frage der Menschenwürde - und kann die Behandlung erleichtern.

Gehörlosensprechstunde, «best practices» für Spitalaufenthalte von Menschen mit einer Behinderung oder Projekte für ein demenzgerechtes Spital lanciert worden. Den Patienten und die Patientin in ihrer individuellen Situation und Verletzlichkeit wahrnehmen: Das gehört für Ärztinnen und Ärzte seit jeher zur täglichen Arbeit. Es ist "personalisierte Medizin", die weit über Gentests hinausgeht und unser Gesundheitswesen erst human macht. 